\title{
Association between Time to First Cigarette and Health-Related Quality of Life of Middle-Aged Male Current Smokers: A Nationwide Representative Study in Korea
}

\author{
Sung Eun Jo ${ }^{1,2}$, Hye Rim Hwang ${ }^{1,2 *}$, Yun Jin Kim ${ }^{1,2}$, Sang Yeoup Lee ${ }^{3,4}$, Jeong Gyu Lee ${ }^{1,2,5}$, Yu Hyeon Yi ${ }^{1,2,5}$, \\ Young Hye Cho ${ }^{3}$, Young Jin Tak ${ }^{1,2}$, Seung Hun Lee ${ }^{1,2,5}$, Eun Ju Park ${ }^{3}$, Youngin Lee ${ }^{3,5}$ \\ 'Department of Family Medicine, Pusan National University School of Medicine, Yangsan, Korea \\ ${ }^{2}$ Medical Research Institute, Pusan National University Hospital, Busan, Korea \\ ${ }^{3}$ Family Medicine Clinic, Obesity, Metabolism and Nutrition Center, Research Institute of Convergence of Biomedical Science and Technology, Pusan \\ National University Yangsan Hospital, Yangsan, Korea \\ ${ }^{4}$ Department of Medical Education, Pusan National University School of Medicine, Yangsan, Korea \\ ${ }^{5}$ Busan Tobacco Control Center, Pusan National University Hospital, Busan, Korea
}

Background: Although many studies have demonstrated that the first cigarette in the morning increases the prevalence of smoking-related morbidity, limited studies have examined the impact of time to first cigarette (TTFC) on the health-related quality of life (HRQoL). Thus, we assessed this relationship using nationally-representative data from the Korea National Health and Nutrition Examination Survey VII-1 (2016).

Methods: We conducted a cross-sectional study using data from 577 current male smokers aged 30-59 years, after excluding those with a certain disease. Participants were divided into four categories according to TTFC $(\leq 5 \mathrm{~min}$, 6-30 min, 31-60 min, >60 min). HRQoL was measured using self-reported EuroQol-5 (EQ-5D). The relationship between TTFC and EQ-5D index was analyzed using a multivariate-adjusted generalized linear model to assess how HRQoL varies according to TTFC. After adjusting for confounders, a multivariate-adjusted logistic regression analysis was performed to identify which of the five dimensions of the EQ-5D affected the HRQoL according to TTFC.

Results: The generalized linear analysis indicated that as TTFC decreased (6-30 $\mathrm{min}, 31-60 \mathrm{~min}$ vs. $>60 \mathrm{~min}$ ), the EQ-5D index score decreased significantly $(\mathrm{P}=0.037)$. Shorter TTFC ( $\leq 5 \mathrm{~min}$ vs. $>60 \mathrm{~min}$ ) was associated with higher pain/discomfort (odds ratio [OR], 3.82; 95\% confidence interval [CI], 1.39-10.48) and anxiety/depression (OR, $7.58 ; 95 \%$ CI, 1.75-32.88).

Conclusion: Higher nicotine dependence was associated with impaired HRQoL. These results may be used to improve smoking cessation treatment outcomes.

Keywords: Smoking; Quality of Life

Received: August 29, 2019, Revised: April 19, 2020, Accepted: April 23, 2020

${ }^{*}$ Corresponding Author: Hye Rim Hwang https://orcid.org/0000-0001-7658-3749

Tel: +82-51-240-7834, Fax: +82-51-240-7843, E-mail: hezera83@naver.com 


\section{INTRODUCTION}

Cigarette smoking is a health-threatening behavior that not only increases the complications of cardiovascular and respiratory diseases but also has a causal relationship with other cancers, such as cancer of the lung, oral cavity, larynx, pharynx, esophagus, stomach, bladder, kidney, and pancreas. ${ }^{1)}$ It is also one of the leading preventable causes of deaths worldwide. An estimated 100 million people died due to tobacco-related diseases in the 20th century. This figure is expected to increase by 10 times through the 21 st century. ${ }^{2)}$

Recent studies have shown that smoking could be associated with other health outcomes, including a poor health-related quality of life (HRQoL) ${ }^{3)}$ HRQoL is a useful indicator of the health status of a population and could be used to evaluate the performance of the health system. ${ }^{4}$ Previous studies that assessed the quality of life of smokers identified an inverse relationship between smoking and quality of life. ${ }^{1)}$ Although several studies have examined the associations between smoking and HRQOL, limited studies have investigated the effect of nicotine dependence on HRQOL of current smokers. Nicotine addiction can be measured using the Fagerstrom Test for Nicotine Dependence (FTND), and time to first cigarette (TTFC), which is one of the six measures of FTND. ${ }^{5)}$ Many studies have shown that TTFC is a powerful indicator of nicotine dependence. ${ }^{6)}$ Recent studies have reported that early TTFC is associated with hypertension, ${ }^{7)}$ dyslipidemia, ${ }^{8)}$ chronic obstructive pulmonary disease, ${ }^{9)}$ and smoking-related cancers, such as head and neck cancer ${ }^{10)}$ and lung cancer. ${ }^{11)}$ Thus, the purpose of this study was to investigate the relationship between TTFC and HRQoL among Korean adult smokers. We hypothesized that shorter TTFC was associated with a poor HRQoL. That is, we speculated that nicotine dependence is associated not only with morbidity but also poor daily functioning and overall well-being. These findings from our study can be used to encourage individuals to quit smoking.

\section{METHODS}

\section{Participants}

We used data from the Korea National Health and Nutrition Survey (KNHANES) VII-1 conducted in 2016. The survey used a stratified, multilevel probability sample design for the population of South Korea. The KNHANES VII-1 extracted data from 23 households in 192 districts as a probability sample and examined about 10,000 household members including members 1-year-old or older by applying survey items according to life cycle characteristics.

Of 8,150 participants in the KNHANES VII-1 (2016), we included 1,125 current smokers, after excluding non-smokers or ex-smokers or those who did not provide information about their smoking status. Since diseases such as osteoarthritis, ${ }^{12)}$ rheumatoid arthritis, ${ }^{13)}$ cancer, $^{14)}$ and depression ${ }^{15)}$ have been shown to lower HRQoL in several studies, patients with these diseases were excluded. Thus, participants with diseases included 40 cases of osteoarthritis, 10 cases of rheumatoid arthritis, seven cases of cancer, and 27 cases of depression. The seven cases of cancer included one gastric cancer, one colorectal cancer, one lung cancer, and four thyroid cancer patients. Previous studies have shown that nicotine dependence increases until the 50s, after which it starts declining. ${ }^{16)}$ We wanted to examine the relationship between nicotine dependence and HRQoL in the age group where nicotine dependence increases. The participants were limited to ages 30 to 59. Previous studies have shown that nicotine dependence may vary according to gender, ${ }^{17)}$ and since the ratio of female smokers in this data is significantly smaller than that of males, only male smokers were included for the accurate analysis. The final sample included 577 current smokers aged 30-59 years without any certain diseases (Figure 1).

The survey was conducted in accordance with the Helsinki Declaration. As the KNHANES datasets do not include identifiable personal information, studies using these data do not require approval from an institutional review board. Applications for exemption from approval have also been implemented (IRB no., H-1907-013-080).

\section{Measures}

\section{1) Measurement of the HRQoL}

HRQoL was assessed using the EuroQol-5 (EQ-5D) dimensions, which include mobility, self-care, usual activities, pain/discomfort, and anxi-

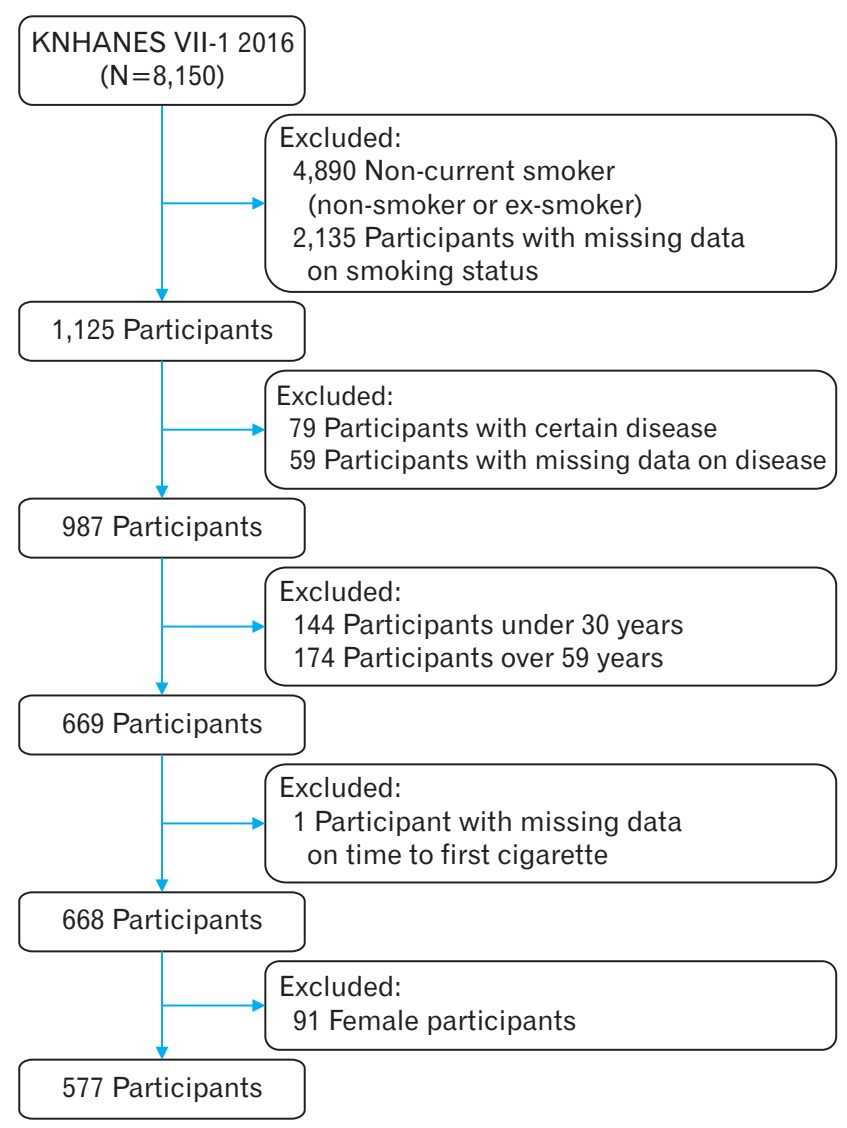

Figure 1. Flow diagram of the study participants. KNHANES, Korea National Health and Nutrition Survey. 
ety/depression. Each dimension is evaluated in three levels ("no problem," "some problems," and "extreme/severe problems"). Answers to each dimension can be combined into 243 health states. ${ }^{18)}$ A combination of these items is used to calculate the health index score (EQ-5D index) using the Korean evaluation set developed by the Korea Centers for Disease Control and Prevention. The EQ-5D index is calculated as the weighted index value from complete health status 1 to the lowest score $-0.171 .{ }^{19)}$ We defined "some problems" or "extreme/severe problems" as "problematic" in each dimension of the EQ-5D.

\section{2) TTFC and smoking behavior}

TTFC was first examined in the KNHANES VII-1. It was evaluated us- ing the question, "How soon do you smoke first in the morning?" with four possible response choices ( $\leq 5$ minutes, 6-30 minutes, 31-60 minutes, and $>60$ minutes). Smoking behavior was assessed by self-report items on the age of initial smoking and the amount of cigarette smoking per day (CPD). Smoking duration (years) was calculated by subtracting the age of initial smoking from the current age. The pack-years of cigarette smoking were calculated as the average number of cigarette packs smoked per day multiplied by the number of years of daily smoking. ${ }^{7)}$

\section{3) Covariates}

Data on participants' socio-demographic characteristics, physical ac-

Table 1. General characteristics of the participants according to TTFC

\begin{tabular}{|c|c|c|c|c|c|}
\hline \multirow{2}{*}{ Characteristic } & \multicolumn{4}{|c|}{ TTFC } & \multirow{2}{*}{ P-value } \\
\hline & $\leq 5 \min (n=122)$ & $6-30 \min (n=189)$ & $31-60 \min (n=102)$ & $>60 \min (n=164)$ & \\
\hline Age $(y)$ & $43.22 \pm 0.77$ & $43.90 \pm 0.63$ & $41.73 \pm 1.01$ & $44.65 \pm 0.76$ & 0.083 \\
\hline Economic status: employed ${ }^{*}$ & $83.1 \pm 4.1(104)$ & $92.7 \pm 2.0(173)$ & $94.6 \pm 2.4(97)$ & $90.3 \pm 1.4(522)$ & 0.430 \\
\hline Individual income & & & & & 0.009 \\
\hline Lowest & $38.0 \pm 5.8(51)$ & $30.7 \pm 4.4(54)$ & $25.2 \pm 6.0(24)$ & $16.7 \pm 3.4(26)$ & \\
\hline Lower-middle & $23.1 \pm 4.4(25)$ & $29.8 \pm 3.5(61)$ & $21.9 \pm 4.5(22)$ & $22.8 \pm 3.2(41)$ & \\
\hline Upper-middle & $17.0 \pm 3.7(20)$ & $19.9 \pm 3.5(37)$ & $30.8 \pm 5.2(34)$ & $28.6 \pm 6.3(45)$ & \\
\hline Highest & $21.9 \pm .4 .1(26)$ & $19.6 \pm 2.9(37)$ & $22.1 \pm 4.6(22)$ & $31.9 \pm 4.2(51)$ & \\
\hline \multicolumn{6}{|l|}{ Level of education } \\
\hline Elementary school & $5.4 \pm 1.8(9)$ & $6.1 \pm 1.9(10)$ & $0.9 \pm 0.8(1)$ & $4.1 \pm 1.8(6)$ & $<0.001$ \\
\hline Middle school & $13.0 \pm 3.2(16)$ & $7.4 \pm 2.4(14)$ & $9.8 \pm 3.4(9)$ & $7.4 \pm 2.3(11)$ & \\
\hline High school & $43.8 \pm 4.7(52)$ & $43.5 \pm 3.8(77)$ & $28.7 \pm 5.2(29)$ & $22.6 \pm 3.2(41)$ & \\
\hline University & $37.8 \pm 4.6(45)$ & $43.0 \pm 4.2(86)$ & $60.7 \pm 5.4(63)$ & $65.9 \pm 4.4(105)$ & \\
\hline \multicolumn{6}{|l|}{ Marital status } \\
\hline Single & $35.9 \pm 5.5(40)$ & $15.1 \pm 3.3(24)$ & $12.9 \pm 3.6(12)$ & $9.0 \pm 2.3(15)$ & $<0.001$ \\
\hline Living with spouse & $56.1 \pm 5.9(72)$ & $77.9 \pm 4.1(153)$ & $87.1 \pm 3.6(90)$ & $84.4 \pm 3.3(139)$ & \\
\hline Separated/bereavement/divorced & $8.0 \pm 2.8(10)$ & $7.0 \pm 2.1(12)$ & 0 & $6.6 \pm 2.3(10)$ & \\
\hline Hospital visit $^{\dagger}$ & $25.2 \pm 4.0(29)$ & $18.3 \pm 3.0(35)$ & $25.0 \pm 5.2(24)$ & $23.5 \pm 3.7(34)$ & 0.506 \\
\hline Aerobic physical activity ${ }^{\ddagger}$ & $41.4 \pm 4.8(43)$ & $39.7 \pm 3.8(76)$ & $40.9 \pm 6.2(42)$ & $57.2 \pm 4.3(89)$ & 0.019 \\
\hline Stress & & & & & 0.211 \\
\hline Low & $55.6 \pm 5.2(70)$ & $67.2 \pm 3.7(119)$ & $56.2 \pm 5.6(62)$ & $63.6 \pm 4.4(104)$ & \\
\hline High & $44.4 \pm 5.2(52)$ & $32.8 \pm 3.7(70)$ & $43.8 \pm 5.6(40)$ & $36.4 \pm 4.4(60)$ & \\
\hline High-risk drinking ${ }^{\S}$ & $42.5 \pm 4.6(51)$ & $35.7 \pm 3.6(68)$ & $32.4 \pm 5.1(32)$ & $27.9 \pm 4.1(45)$ & 0.110 \\
\hline Age of first cigarette (y) & $17.77 \pm 0.20$ & $18.45 \pm 0.26$ & $18.85 \pm 0.32$ & $19.90 \pm 0.24$ & $<0.001$ \\
\hline Smoking (y) & $25.45 \pm 0.74$ & $25.45 \pm 0.61$ & $22.88 \pm 1.04$ & $24.75 \pm 0.76$ & 0.150 \\
\hline Cigarettes per day (d) & $20.18 \pm 0.88$ & $16.49 \pm 0.54$ & $14.24 \pm 0.73$ & $9.45 \pm 0.52$ & $<0.001$ \\
\hline Smoking (pack-years) & $26.08 \pm 1.20$ & $21.63 \pm 1.05$ & $17.20 \pm 1.41$ & $12.32 \pm 0.85$ & $<0.001$ \\
\hline EQ-5D index & $0.961 \pm 0.008$ & $0.963 \pm 0.007$ & $0.971 \pm 0.008$ & $0.983 \pm 0.004$ & 0.011 \\
\hline \multicolumn{6}{|l|}{$E Q-5 D$} \\
\hline Mobility & $10.1 \pm 3.5(10)$ & $7.0 \pm 2.0(13)$ & $4.3 \pm 2.2(4)$ & $4.4 \pm 1.6(8)$ & 0.305 \\
\hline Self-care & $0.6 \pm 0.6(1)$ & $1.8 \pm 2.9(4)$ & $1.7 \pm 1.2(2)$ & $0.8 \pm 0.6(2)$ & 0.636 \\
\hline Usual activity & $4.2 \pm 2.9(4)$ & $4.0 \pm 1.6(8)$ & $1.7 \pm 1.2(2)$ & $1.7 \pm 0.9(4)$ & 0.515 \\
\hline Pain/discomfort & $22.1 \pm 4.5(24)$ & $18.2 \pm 3.0(32)$ & $13.3 \pm 3.8(12)$ & $9.4 \pm 2.6(15)$ & 0.047 \\
\hline Anxiety/depression & $9.6 \pm 2.6(12)$ & $7.0 \pm 2.1$ (13) & $8.7 \pm 4.0(5)$ & $2.9 \pm 1.3(35)$ & 0.156 \\
\hline
\end{tabular}

Values are presented as estimated mean $\% \pm S D$ (unweighted number) for categorical variables or estimated mean $\pm S D$ for continuous variables. P-values were obtained by Pearson's chi-square test for categorical variables and general linear model analysis for continuous variables. Bold type is considered statistically significant.

TTFC, time to first cigarette; SD, standard deviation; EQ-5D, EuroQol-5 dimensions.

${ }^{*}$ Economic status of participants was classified as employed, unemployed, and non-economically active population. ${ }^{\dagger}$ Hospital visits were surveyed by asking if they had been treated in an outpatient clinic within the last 2 weeks. ${ }^{\star}$ Aerobic physical activity was defined as one of the following: (1) more than 2 hours and 30 minutes of medium intensity physical activity per week, (2) more than 1 hour and 15 minutes of high intensity physical activity per week, (3) Combining mid- and high-intensity physical activities (a

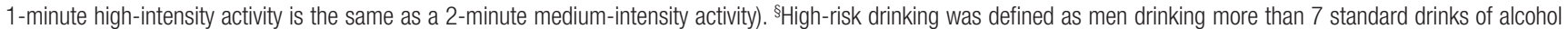
per day more than twice per week and women drinking more than 5 standard drinks of alcohol per day more than twice per week. 
tivity, and mental status were obtained. We defined high-risk drinking as more than seven standard drinks of alcohol per day for more than twice per week for men and more than five standard drinks of alcohol per day for more than twice per week for women. ${ }^{7)}$ Aerobic physical activity was defined as one of the following: (1) more than 2 hours and 30 minutes of medium-intensity physical activity per week, (2) more than 1 hour and 15 minutes of high-intensity physical activity per week, (3) combining mid- and high-intensity physical activity (a 1-minute high-intensity activity was considered the same as a 2-minute medium-intensity activity). ${ }^{7}$ Participants' economic status was classified as employed, unemployed, and non-economically active population. Monthly personal income was classified into four categories: lowest, lower-middle, upper-middle, or highest. The degree of stress was assessed using the question, "How much stress do you usually experience in your daily life?" Participants responded with four items with "I feel a lot," "I feel moderate," "I feel a little," and "I feel very little." Participants who responded with "I feel a lot" and "I feel moderate" were defined as "highly stressed." Education levels were classified into four categories: elementary school graduation, middle school graduation, high school graduation, and university graduation. Current marital status was classified into single, living with a spouse, and separated/bereavement/divorced. The recent hospital visits were surveyed by asking if they had been treated in an outpatient clinic within the last 2 weeks.

\section{Statistical Analyses}

We compared the differences in participants' characteristics according to their TTFC. The categorical variables were analyzed using Pearson's chi-square test, and the continuous variables were analyzed using generalized linear models. Data for continuous variables are presented as means \pm standard deviation, and those for categorical variables are presented as means \pm standard deviation (\%, unweighted number). A multivariate-adjusted general linear analysis was subsequently performed to estimate how the HRQoL varies according to the TTFC. We calculated the mean difference and its $95 \%$ confidence interval (CI) in the EQ-5D index for each TTFC category ( $\leq 5$ minutes, $6-30$ minutes, 31-60 minutes, and $>60$ minutes) using TTFC $>60$ minutes as refer- ence. Finally, a logistic regression analysis was performed to identify which of the five dimensions of the EQ-5D had a negative effect on HRQoL as the TTFC decreased. The logistic regression analysis was performed after adjusting multivariate with "problematic" as a dependent variable for each item of EQ-5D. Analyses were adjusted for age, smoking behavior (smoking pack-years), economic status, individual income, education level, marital status, hospital visit, stress level, regular exercise, and high-risk drinking. All analyses were performed using IBM SPSS ver. 21.0 (IBM Corp., Armonk, NY, USA). A P-value $<0.05$ was considered statistically significant.

\section{RESULTS}

\section{General Characteristics of the Participants according to Their Time to First Cigarette}

Characteristics of participants according to the TTFC are presented in Table 1. We confirmed that there were significant differences between each group according to TTFC in individual income $(\mathrm{P}=0.009)$, level of education $(\mathrm{P}<0.001)$, marital status $(\mathrm{P}<0.001)$, and aerobic physical activity ( $\mathrm{P}=0.019)$. Moreover, we also found significant differences between each group by TTFC in the age of first cigarette $(\mathrm{P}<0.001)$, CPD $(\mathrm{P}<0.001)$, and smoking pack-years $(\mathrm{P}<0.001)$. In the EQ-5D index $(\mathrm{P}=0.011)$, there was a significant difference between each group according to TTFC. However, in each of the EQ-5D items, only pain/discomfort $(\mathrm{P}=0.047)$ showed a significant difference according to TTFC.

\section{Association between Health-Related Quality of Life and Time to First Cigarette}

The association between TTFC and HRQoL was examined without adjustment (model 1); with adjusting for age (model 2); with adjusting for age, and smoking behaviors (smoking pack-years) (model 3); and with adjusting for age, smoking behaviors (smoking pack-years), socioeconomic status, and health-related behaviors (model 4). Table 2 presents the results of the multivariate-adjusted general linear analysis of the mean differences in the EQ-5D indexes between the TTFC $>60$ minutes group and the remaining groups. In the fully adjusted model, the mean EQ-5D index of the 5 minutes $<$ TTFC $\leq 30$ minutes group was

Table 2. Multivariate adjusted generalized linear model according to TTFC

\begin{tabular}{|c|c|c|c|c|}
\hline \multirow{2}{*}{ TTFC } & \multicolumn{4}{|c|}{ Mean difference of EQ-5D index } \\
\hline & Model 1 & Model 2 & Model 3 & Model 4 \\
\hline$\leq 5 \min$ vs. $>60 \mathrm{~min}$ & $-0.022^{*}(-0.040$ to -0.004$)$ & $-0.023^{*}(-0.041$ to -0.005$)$ & $-0.030^{*}(-0.053$ to -0.008$)$ & $-0.014(-0.035$ to 0.007$)$ \\
\hline $6-30$ min vs. $>60 \mathrm{~min}$ & $-0.020^{*}(-0.035$ to -0.004$)$ & $-0.020^{*}(-0.036$ to -0.004$)$ & $-0.025^{\star}(-0.042$ to -0.008$)$ & $-0.022^{*}(-0.037$ to -0.006$)$ \\
\hline $31-60 \mathrm{~min}$ vs. $>60 \mathrm{~min}$ & $-0.012(-0.029$ to 0.004$)$ & $-0.014(-0.031$ to 0.002$)$ & $-0.018^{*}(-0.035$ to -0.001$)$ & $-0.015^{\star}(-0.030$ to 0.000$)$ \\
\hline P-value & 0.012 & 0.008 & 0.007 & 0.037 \\
\hline$P$ for trend & 0.005 & 0.004 & 0.005 & 0.086 \\
\hline
\end{tabular}

Values are presented as mean differences $(95 \% \mathrm{Cl})$ in the $\mathrm{EQ}-5 \mathrm{D}$ index. Odds ratios and $95 \% \mathrm{Cl}$ were estimated by multivariate adjusted generalized linear analyses. Model 1 : crude; model 2: adjusted for age; model 3: adjusted for age and smoking behaviors (smoking pack-years); model 4: adjusted for age, smoking behaviors (smoking packyears), individual income, economic status, marital status, education level, hospital visit, aerobic physical activities, stress, and high-risk drinking. Bold type is considered statistically significant.

TTFC, time to first cigarette; EQ-5D, EuroQol-5 dimensions; Cl, confidence interval.

${ }^{*} \mathrm{P}<0.05$. 
significantly lower than that of the TTFC $>60$ minutes group, and the 30 minutes $<$ TTFC $\leq 60$ minutes group also obtained the same result.

Among the five items of EQ-5D, shorter TTFC was associated with higher pain/discomfort and anxiety/depression, and this association was significant after adjusting for various confounders. As shown in model 4 in Table 3, the odds ratios were 2.14 (95\% CI, 0.90-5.09), 3.66 (95\% CI, 1.59-8.38), and 3.82 (95\% CI, 1.39-10.48) at 31-60 minutes, 6-30 minutes, and $\leq 5$ minutes, respectively, compared to the TTFC $>60$ minutes group in the pain/discomfort dimension ( $\mathrm{P}$ for trend= 0.006). For the anxiety/depression dimension, the odds ratios were 6.04 (95\% CI, 1.21-30.10), 4.64 (95\% CI, 1.19-18.16), and 7.58 (95\% CI, $1.75-32.88$ ) at 31-60 minutes, $6-30$ minutes, and $\leq 5$ minutes, respectively, compared to the TTFC $>60$ minutes ( $\mathrm{P}$ for trend $=0.010$ ).

\section{DISCUSSION}

This study examined the association between TTFC and HRQoL among 577 current male smokers in a nationally representative sample of Korean adults aged 30-59 years. We found that shorter TTFC was associated with poor HRQoL after adjusting for other possible factors associated with smoking status and HRQoL.

Self-reported health status is an important indicator of morbidity and a stronger predictor of quality of life outcomes compared to other morbidity measures. ${ }^{20)}$ Moreover, self-report data collection is more cost-effective and can be more comprehensive compared to medical record reviews. ${ }^{21)}$ Much of the predictive cogency of FTND may be due to its first item, i.e., TTFC, which is more valid than any other single measure. ${ }^{22)}$ Thus, among the current smokers with high nicotine dependence, which can be identified with shorter TTFC, poor HRQoL could be a useful predictor of smoking-related morbidity. ${ }^{6)}$

Limited studies have investigated the effects of TTFC on self-reported health. Our findings on the relationship between TTFC and HRQoL are consistent with those of one previous study that used data from the National Adult Tobacco Surveys in the United States. ${ }^{6)}$ However, in the previous study, health status was evaluated using the item, "In general, you would consider your health to be" with five possible answers (i.e., excellent, very good, good, fair, poor). Therefore, it was difficult to directly compare the results of the EQ-5D. To our knowledge, this study is the first to investigate the association between TTFC and HRQoL using EQ-5D.

In our study, except for the $\leq 5$ minutes group, EQ- $5 \mathrm{D}$ index decreased as the TTFC became shorter in the fully adjusted model, which means that the shorter the TTFC, the lower the HRQoL. The logistic regression model identified which of the five items in EQ-5D had

Table 3. OR and $95 \% \mathrm{Cl}$ for impaired health-related quality of life according to TTFC

\begin{tabular}{|c|c|c|c|c|c|c|}
\hline \multirow{2}{*}{ Model } & \multirow{2}{*}{ Category } & \multicolumn{4}{|c|}{ TTFC } & \multirow{2}{*}{ P for trend } \\
\hline & & $>60$ min & $31-60 \mathrm{~min}$ & $6-30 \mathrm{~min}$ & $\leq 5 \min$ & \\
\hline \multirow[t]{6}{*}{ Model 1} & Dimensional problem of EQ-5D & & & & & \\
\hline & Mobility & 1 & $0.97(0.25-3.72)$ & $1.62(0.64-4.13)$ & $2.41(0.86-6.74)$ & 0.075 \\
\hline & Self-care & 1 & $2.22(0.29-16.51)$ & $2.32(0.37-14.53)$ & $0.80(0.67-9.62)$ & 0.916 \\
\hline & Usual activity & 1 & $1.03(0.18-5.87)$ & $2.40(0.66-8.65)$ & $2.57(0.41-16.08)$ & 0.216 \\
\hline & Pain/discomfort & 1 & $1.48(0.65-3.38)$ & $2.14^{\star}(1.06-4.32)$ & $2.73^{*}(1.24-6.03)$ & 0.008 \\
\hline & Anxiety/depression & 1 & $3.24(0.84-12.53)$ & $2.54(0.89-7.23)$ & $3.62^{*}(1.20-10.91)$ & 0.042 \\
\hline \multirow[t]{6}{*}{ Model 2} & Dimensional problem of EQ-5D & & & & & \\
\hline & Mobility & 1 & $1.17(0.31-4.47)$ & $1.74(0.67-4.51)$ & $2.72(0.93-7.91)$ & 0.060 \\
\hline & Self-care & 1 & $3.17(0.40-25.41)$ & $2.67(0.41-17.82)$ & $1.02(0.08-13.36)$ & 0.745 \\
\hline & Usual activity & 1 & $1.30(0.22-7.52)$ & $2.61(0.70-9.70)$ & $2.98(0.46-19.40)$ & 0.180 \\
\hline & Pain/discomfort & 1 & $1.64(0.72-3.74)$ & $2.21^{*}(1.08-4.53)$ & $2.89^{\star}(1.30-6.43)$ & 0.006 \\
\hline & Anxiety/depression & 1 & $3.27(0.87-12.33)$ & $2.55(0.89-7.28)$ & $3.64^{*}(1.19-11.16)$ & 0.045 \\
\hline \multirow[t]{6}{*}{ Model 3} & Dimensional problem of EQ-5D & & & & & \\
\hline & Mobility & 1 & $1.16(0.29-4.67)$ & $1.71(0.58-5.04)$ & $2.66(0.63-11.20)$ & 0.160 \\
\hline & Self-care & 1 & $5.09(0.58-45.02)$ & $5.10(0.79-32.76)$ & $2.69(0.26-27.79)$ & 0.099 \\
\hline & Usual activity & 1 & $1.95(0.29-13.08)$ & $4.50(1.14-17.68)$ & $6.80(0.65-71.54)$ & 0.063 \\
\hline & Pain/discomfort & 1 & $1.99(0.87-4.59)$ & $2.87^{\star}(1.34-6.16)$ & $4.28^{\star}(1.70-10.75)$ & 0.002 \\
\hline & Anxiety/depression & 1 & $4.33(0.98-19.07)$ & $3.69^{*}(1.18-11.52)$ & $6.38^{*}(1.44-28.26)$ & 0.017 \\
\hline \multirow[t]{6}{*}{ Model 4} & Dimensional problem of EQ-5D & & & & & \\
\hline & Mobility & 1 & $0.76(0.14-4.05)$ & $1.37(0.34-5.54)$ & $1.22(0.24-6.04)$ & 0.696 \\
\hline & Self-care & 1 & $28.72(0.84-9794.46)$ & $6.28(0.12-317.53)$ & $3.18(0.05-207.60)$ & 0.987 \\
\hline & Usual activity & 1 & $2.31(0.21-25.16)$ & $9.38(0.85-103.21)$ & $6.24(0.34-114.83)$ & 0.173 \\
\hline & Pain/discomfort & 1 & $2.14(0.90-5.09)$ & $3.66^{*}(1.59-8.38)$ & $3.82 *(1.39-10.48)$ & 0.006 \\
\hline & Anxiety/depression & 1 & $6.04^{*}(1.21-30.10)$ & $4.64^{*}(1.19-18.16)$ & $7.58^{\star}(1.75-32.88)$ & 0.010 \\
\hline
\end{tabular}

Values are presented as OR $(95 \% \mathrm{Cl})$. ORs and $95 \% \mathrm{Cl}$ were estimated by multivariate adjusted logistic regression analyses. Model 1: crude; model 2: adjusted for age; model 3: adjusted for age and smoking behaviors (smoking pack-years); model 4: adjusted for age, smoking behaviors (smoking pack-years), individual income, economic status, marital status, education level, hospital visit, aerobic physical activities, stress, and high-risk drinking. Bold type is considered statistically significant.

$\mathrm{OR}$, odds ratio; $\mathrm{Cl}$, confidence interval; TTFC, time to first cigarette; EQ-5D, EuroQol-5 dimensions.

${ }^{*} \mathrm{P}<0.05$. 
a significant effect on reducing HRQoL as TTFC decreased. Among the five items of the EQ-5D, shorter TTFC was associated with higher pain/discomfort and anxiety/depression, and it was significant after adjusting for various confounders.

Similar to our study, one previous study found that smokers with high nicotine dependence, defined as the first tobacco smoking group within 30 minutes of awakening, exhibited a particularly high rate of depressive symptoms. ${ }^{23)}$ Several studies have reported that nicotine might be a pharmacologically active ingredient in tobacco smoking, and it is known to have direct and indirect effects on neurotransmitters related to major depressive disorder. ${ }^{23,24)}$ A study using an animal model showed that nicotine exposure does not regulate the hypothalamus-pituitary-adrenal system, causing cortisol hyper-administration and altering the activity of the relevant monoamine neurotransmitter system. This function regulates the response to stressors, which is normalized after nicotine elimination. ${ }^{25)}$

Furthermore, several studies have reported that nicotine leads to neuronal activation and hypersensitivity, causing pain, ${ }^{26)}$ and elevated nicotine levels also promote changes in the cells related to the peripheral and central nociceptor sensitization. ${ }^{27)}$ One previous study showed that patients who experienced pain and are diagnosed with various pathologies and those with nicotine dependence had a higher degree of pain, which interfered with their daily life and mood. Patients with chronic tobacco use and those with severe nicotine dependence who experienced pain had significantly higher pain intensities compared to non-users. $^{28)}$

Another previous study reported that cotinine is a metabolite of nicotine, and TTFC is a strong predictor of nicotine intake because shorter TTFC increases blood and urine cotinine levels. ${ }^{29)}$ Thus, it can be suggested that the shorter the TTFC, the stronger the action of nicotine against depression/anxiety and pain/discomfort. Based on these findings, it can be suggested that HRQoL deteriorates with shorter TTFC.

However, several studies have reported that baseline depression or anxiety may also be related to a type of subsequent smoking behavior, such as the onset of smoking itself, excessive smoking, or the shift from daily smoking to nicotine dependence. These bidirectional pathways of presumption are not mutually exclusive; however, a few studies have reported evidence for a bidirectional association between smoking and depression/anxiety. ${ }^{25)}$ Therefore, further research using different methods is required to confirm their causal relationship. Another study reported that individuals with chronic pain not medically described were more likely to become current smokers and nicotine dependents than were those without chronic pain. It is unclear whether chronic pain affects nicotine administration, but it was explained that smokers with chronic pain could induce smoking to alleviate discomfort or somatosensory conditions. ${ }^{30)}$ Therefore, further studies are needed to confirm the causal relationship between these factors.

The principal strength of our study was its use of a large-scale, nationally representative sample of middle-aged Korean male adults. We also adjusted for potential confounding factors that could affect the results.
However, this study has several limitations. First, the cross-sectional design of the study precludes any causal inferences about the relationship between TTFC and HRQoL. Therefore, longitudinal studies are necessary to examine a causal link between these two. Second, the sample consisted of respondents from South Korea alone. Since nicotine metabolism and smoking behaviors may vary across races, further studies of smokers from other racial groups and geographical areas should be conducted. Third, subjects were limited to middle-aged male current smokers. Therefore, further studies are needed to confirm whether nicotine dependence and HRQoL have a significant relationship, regardless of age, or only in certain age groups. Fourth, we did not consider mental disorders related to both nicotine dependence and HRQoL in this study. Thus, further studies must analyze the mental disorders related to nicotine dependence and HRQoL. Finally, we adjusted for various confounders in this study, but the possibility of residual confounders cannot be ruled out.

In conclusion, earlier TTFC is associated with higher depression/ anxiety and pain/discomfort, which may lead to the deterioration of HRQoL. In other words, it not only increases morbidity but also reduces daily activities and overall well-being due to nicotine dependence. These findings could be used to motivate participants to quit smoking. The results of this study also suggest that smokers with poor HRQoL may not be able to control their nicotine dependence. In addition, physicians should provide mood control and pain management interventions in smoking cessation treatments to improve the HRQoL of smokers with high nicotine dependence.

\section{CONFLICT OF INTEREST}

No potential conflict of interest relevant to this article was reported.

\section{ORCID}

Sung Eun Jo: https://orcid.org/0000-0001-6469-0942

Hye Rim Hwang: https://orcid.org/0000-0001-7658-3749

Yun Jin Kim: https://orcid.org/0000-0002-0204-3253

Sang Yeoup Lee: https://orcid.org/0000-0002-3585-9910

Jeong Gyu Lee: https://orcid.org/0000-0001-7160-0714

Yu Hyeon Yi: https://orcid.org/0000-0002-1786-2737

Young Hye Cho: https://orcid.org/0000-0003-2176-6227

Young Jin Tak: https://orcid.org/0000-0002-4645-5866

Seung Hun Lee: https://orcid.org/0000-0002-0976-8708

Eun Ju Park: https://orcid.org/0000-0003-2415-8243

Youngin Lee: https://orcid.org/0000-0003-0141-7484

\section{REFERENCES}

1. Davila EP, Zhao W, Byrne M, Hooper MW, Messiah A, Caban-Martinez A, et al. Health-related quality of life and nicotine dependence, Florida 2007. Am J Health Behav 2011;35:280-9.

2. White WB. Smoking-related morbidity and mortality in the cardiovas- 
cular setting. Prev Cardiol 2007;10:1-4.

3. McClave AK, Dube SR, Strine TW, Mokdad AH. Associations between health-related quality of life and smoking status among a large sample of U.S. adults. Prev Med 2009;48:173-9.

4. Romero M, Vivas-Consuelo D, Alvis-Guzman N. Is health related quality of life (HRQoL) a valid indicator for health systems evaluation? Springerplus 2013;2:664.

5. Ahn HK, Lee HJ, Jung DS, Lee SY, Kim SW, Kang JH. The reliability and validity of Korean version of questionnaire for nicotine dependence. J Korean Acad Fam Med 2002;23:999-1008.

6. Sung B. Time to first cigarette and self-reported health among US adult smokers. Tob Use Insights 2019;12:1179173X18825262.

7. Bae J, Yi YH, Kim YJ, Lee JG, Tak YJ, Lee SH, et al. Time to first cigarette and the risk of hypertension: a nationwide representative study in Korea. Am J Hypertens 2019;32:202-8.

8. Selya AS, Hesse ND. Time to first cigarette and serum cholesterol levels. Soc Sci Med 2017;174:213-9.

9. Kim G, Song H, Park K, Noh H, Lee E, Lee H, et al. Association of time to first morning cigarette and chronic obstructive pulmonary disease measured by spirometry in current smokers. Korean J Fam Med 2018; 39:67-73.

10. Muscat JE, Ahn K, Richie JP Jr, Stellman SD. Nicotine dependence phenotype, time to first cigarette, and risk of head and neck cancer. Cancer 2011;117:5377-82.

11. Gu F, Cheung LC, Freedman ND, Katki HA, Caporaso NE. Potential impact of including time to first cigarette in risk models for selecting ever-smokers for lung cancer screening. J Thorac Oncol 2017;12:164653.

12. Vennu V, Bindawas SM. Relationship between falls, knee osteoarthritis, and health-related quality of life: data from the Osteoarthritis Initiative study. Clin Interv Aging 2014;9:793-800.

13. Bedi GS, Gupta N, Handa R, Pal H, Pandey RM. Quality of life in Indian patients with rheumatoid arthritis. Qual Life Res 2005;14:1953-8.

14. Smith AW, Bellizzi KM, Keegan TH, Zebrack B, Chen VW, Neale AV, et al. Health-related quality of life of adolescent and young adult patients with cancer in the United States: the adolescent and young adult health outcomes and patient experience study. J Clin Oncol 2013;31: 2136-45.

15. Daly EJ, Trivedi MH, Wisniewski SR, Nierenberg AA, Gaynes BN, Warden D, et al. Health-related quality of life in depression: a STAR*D report. Ann Clin Psychiatry 2010;22:43-55.

16. Park S, Lee JY, Song TM, Cho SI. Age-associated changes in nicotine dependence. Public Health 2012;126:482-9.
17. Pogun S, Yararbas G. Sex differences in nicotine action. In: Henningfield JE, London ED, Pogun S, editors. Nicotine psychopharmacology. Berlin: Springer; 2009. p. 261-91.

18. Rabin R, de Charro F. EQ-5D: a measure of health status from the EuroQol Group. Ann Med 2001;33:337-43.

19. Lee YK, Nam HS, Chuang LH, Kim KY, Yang HK, Kwon IS, et al. South Korean time trade-off values for EQ-5D health states: modeling with observed values for 101 health states. Value Health 2009;12:1187-93.

20. Bayliss EA, Ellis JL, Steiner JF. Seniors' self-reported multimorbidity captured biopsychosocial factors not incorporated into two other data-based morbidity measures. J Clin Epidemiol 2009;62:550-7.

21. Lash TL, Mor V, Wieland D, Ferrucci L, Satariano W, Silliman RA. Methodology, design, and analytic techniques to address measurement of comorbid disease. J Gerontol A Biol Sci Med Sci 2007;62:2815.

22. Transdisciplinary Tobacco Use Research Center (TTURC) Tobacco Dependence, Baker TB, Piper ME, McCarthy DE, Bolt DM, Smith SS, et al. Time to first cigarette in the morning as an index of ability to quit smoking: implications for nicotine dependence. Nicotine Tob Res 2007;9 Suppl 4:S555-70.

23. Son BK, Markovitz JH, Winders S, Smith D. Smoking, nicotine dependence, and depressive symptoms in the CARDIA Study: effects of educational status. Am J Epidemiol 1997;145:110-6.

24. Hall SM, Munoz RF, Reus VI, Sees KL. Nicotine, negative affect, and depression. J Consult Clin Psychol 1993;61:761-7.

25. Fluharty M, Taylor AE, Grabski M, Munafo MR. The association of cigarette smoking with depression and anxiety: a systematic review. Nicotine Tob Res 2017;19:3-13.

26. Liu L, Simon SA. Capsaicin and nicotine both activate a subset of rat trigeminal ganglion neurons. Am J Physiol 1996;270:C1807-14.

27. Hawkins JL, Denson JE, Miley DR, Durham PL. Nicotine stimulates expression of proteins implicated in peripheral and central sensitization. Neuroscience 2015;290:115-25.

28. Weingarten TN, Moeschler SM, Ptaszynski AE, Hooten WM, Beebe TJ, Warner DO. An assessment of the association between smoking status, pain intensity, and functional interference in patients with chronic pain. Pain Physician 2008;11:643-53.

29. Muscat JE, Stellman SD, Caraballo RS, Richie JP Jr. Time to first cigarette after waking predicts cotinine levels. Cancer Epidemiol Biomarkers Prev 2009;18:3415-20.

30. Zvolensky MJ, McMillan K, Gonzalez A, Asmundson GJ. Chronic pain and cigarette smoking and nicotine dependence among a representative sample of adults. Nicotine Tob Res 2009;11:1407-14. 